\title{
Perfectionism and the 'Yips': An Initial Investigation
}

Roberts, R.J.; Rotherham, M.; Maynard, I.; Thomas, O.; Woodman, Tim

\section{Sport Psychologist}

Published: 01/03/2013

Peer reviewed version

Cyswllt i'r cyhoeddiad / Link to publication

Dyfyniad o'r fersiwn a gyhoeddwyd / Citation for published version (APA):

Roberts, R. J., Rotherham, M., Maynard, I., Thomas, O., \& Woodman, T. (2013). Perfectionism and the 'Yips': An Initial Investigation. Sport Psychologist, 27(1), 53-61.

\footnotetext{
Hawliau Cyffredinol / General rights

Copyright and moral rights for the publications made accessible in the public portal are retained by the authors and/or other copyright owners and it is a condition of accessing publications that users recognise and abide by the legal requirements associated with these rights.

- Users may download and print one copy of any publication from the public portal for the purpose of private study or research.

- You may not further distribute the material or use it for any profit-making activity or commercial gain

- You may freely distribute the URL identifying the publication in the public portal ?

Take down policy

as accepted for publication

Take down policy

If you believe that this document breaches copyright please contact us providing details, and we will remove access to the work immediately and investigate your claim.
} 
1 Running Head: PERFECTIONISM AND THE YIPS

2

Article accepted in its current form in The Sport Psychologist:

Roberts, R., Rotheram, M., Maynard, I., Thomas, O., \& Woodman, T. (2013). Perfectionism and the 'yips': An exploratory investigation. The Sport Psychologist, 27, 53-61.

6

7

8

9

10

Perfectionism and the 'yips': An initial investigation

11

Ross Roberts, Mike Rotheram, Ian Maynard, Owen Thomas, \& Tim Woodman

12

13

14

15

16

17

18

19 


\section{Abstract}

21 The present investigation examined whether perfectionism might predict whether an athlete would

22 suffer from the 'yips' (a long-term movement disorder consisting of involuntary movements that

23 affects the execution of motor skills). A sample of 'yips'-affected individuals from golf, cricket, and

24 darts as well as a sport-matched sample of non 'yips'-affected athletes completed the shortened

25 version of Frost, Marten, Lahart, and Rosenblate's (1990) multidimensional perfectionism scale

26 (FMPS). Results revealed that three aspects of perfectionism (personal standards, organization, and

27 concern over mistakes) were associated with a greater likelihood of suffering from the 'yips',

28 indicating that 'yips' sufferers had an unhealthy perfectionism profile. The results highlight

29 perfectionism as a possible antecedent of the 'yips' experience within sport.

30 Keywords: unhealthy perfectionism, perfectionistic striving, perfectionistic concerns, dystonia,

31 choking 


\section{Perfectionism and the 'Yips': An initial investigation}

Within sport, the 'yips' have been defined as a long-term movement disorder consisting of involuntary movements that occur in the course of the execution of finely controlled, skilled motor behaviour (McDaniel, Cummings, \& Shain, 1989). The severity of the 'yips' can be so great that it often results in avoidance-related behaviors such as not wanting to perform the 'yips'-affected task (e.g., Bawden \& Maynard, 2001), or the complete withdrawal from the affected sport (see Smith et al., 2000). While research into this phenomenon has largely focused on golf putting (e.g., McDaniel et al., 1989; Smith et al., 2000; 2003; Stinear et al., 2006), recent findings have indicated that the 'yips' may also be prevalent in other sports including cricket and darts (e.g., Bawden \& Maynard, 2001; Rotheram, Bawden, Maynard, Thomas, \& Scaife, 2006).

Despite this increase in research investigating when and where the 'yips' occur, theoretical explanations of the central causes of the problem remain open to debate. Several authors (e.g., Adler et al., 2005; Sachdev, 1992) suggested that the 'yips' is largely a physical problem that represents a form of focal dystonia (a neurological disorder characterized by involuntary movements, spasms, twisting and posturing of a body part). However, others (e.g., Bawden \& Maynard, 2001) propose that the 'yips' is a psychological problem akin to a severe form of choking. In an attempt to synthesize these disparate views, Smith and colleagues $(2000,2003)$ have proposed a conceptual model whereby the yips are a seen as a performance problem where the underlying mechanisms lie on a continuum between focal dystonia (Type I 'yips') and choking (Type II 'yips'). Thus, in this model, an individual can suffer from the 'yips' as a result of a dystonia or choking. Recent research has provided initial support for this conceptual model by showing that the 'yips' can be caused by dystonia or choking (Stinear et al., 2006).

Notwithstanding researchers' identification of both physiological and psychological mechanisms in the aetiology of the 'yips', anecdotal and empirical research supports a number of common psychological characteristics associated with the phenomenon and those that suffer from 
60 the affliction. For example, Smith et al.'s (2003) model emphasises the central importance of

61 performance anxiety in the development of the 'yips'. However, the precise role of anxiety seems to depend on the type of 'yips' that the performer is suffering from. More specifically, for Type I 'yips', anxiety is suggested to exacerbate the effects of the 'yips' on performance, whereas for Type II 'yips', anxiety is thought to be the central cause (see Smith et al., 2003 for a comprehensive overview). Further to the role of anxiety, 'yips'-affected individuals, particularly in golf, often engage in a number of behavioral modifications (e.g., grip/stance change, use of a different putter) in order to gain some relief from their symptoms and are known to spend a great deal of time obsessing about their problem (Smith et al., 2000; White, 1993). Indeed, qualitative investigations into the 'yips' (e.g., Bawden \& Maynard, 2001; Rotheram, Thomas, Bawden, \& Maynard, 2007) showed that 'yips'-affected individuals engage in obsessive thinking, as the individuals in these studies reported spending considerable time, and investing considerable cognitive resources, in thinking about their problem. In contrast, quantitative evidence (e.g., Adler et al., 2011; McDaniel et al., 1989; Sachdev, 1992) has revealed no significant differences in obsessive thinking between 'yips' affected and non-affected golfers. However, the non-significant differences found in these studies may simply reflect problems with measurement (e.g., measures used were not subject to rigorous psychometric testing and had questionable construct validity). Thus, considering this issue in light of the aforementioned literature indicating that 'yips' affected athletes do appear to engage in obsessive thinking, it seems reasonable to suggest that a 'yips'-obsessive thinking relationship may exist.

In addition to anxiety and obsessive thinking, qualitative investigations into the 'yips'

81 (Bawden \& Maynard, 2001; Rotheram et al., 2006) have reported a number of other psychological characteristics experienced by 'yips'-affected individuals. Such characteristics include: high levels of self-consciousness; perceptions of a lack of control over one's performance; feelings of being 
affected individuals in these studies also reported having recently experienced a significant sportrelated event (e.g., arguments with teammates, playing for a new team) or a major life event (e.g., death of a parent, relationship breakdown) prior to the initial onset of the 'yips', and felt that this event was involved in the first occurrence of the 'yips'. Interestingly, this research also indicates these psychological characteristics are consistent across different sports, despite the fact that the physical symptoms associated with the 'yips' are sport specific (e.g., golfers report involuntary muscle spasms while putting, cricketers and darts players report being unable to release the implement, see Bawden \& Maynard, 2001; Rotheram et al., 2006, 2007; Smith et al., 2003). Given the common psychological characteristics associated with the 'yips' across sports it seems somewhat surprising that research has yet to investigate characteristics that may predispose an individual to suffer from the 'yips', as doing so would help garner a greater understanding of this phenomenon. In this regard, one characteristic that is likely to be relevant to the 'yips' is perfectionism.

Perfectionism is a multi-dimensional construct that is characterized by the setting of extremely high personal standards alongside harsh criticism of one's behavior (Frost, Marten, Lahart, \& Rosenblate, 1990; Hewitt \& Flett, 1991). However, the precise nature of perfectionism remains open to debate amongst researchers. For example, the original conceptualization of perfectionism by Frost and colleagues (1990) contains six dimensions of perfectionism: personal standards, organization, concern over mistakes, doubts about actions, parental expectations, and parental criticism. In contrast, Hewitt and Flett (1991) conceived perfectionism to contain three components: self-oriented perfectionism, socially prescribed perfectionism, and other-oriented perfectionism.

Despite this conceptual debate within the literature, the Frost model remains widely accepted and used (e.g., Cox, Enns, \& Clara, 2002; Frost \& Steketee, 1997; Hall, Kerr, \& Matthews, 1998; Koivula, Hassmén, \& Fallby, 2002). Furthermore, a growing consensus exists that 
110 the relative subcomponents of perfectionism can be incorporated into two broad dimensions:

111 perfectionistic striving, a dimension related to having high personal standards and striving for

112 perfection; and perfectionistic concerns, a dimension related to highly critical self-evaluation (e.g.,

113 see Dunkley, Zureoff, \& Blankstein, 2003; Stoeber \& Otto, 2006). As such, the personal standards

114 and organization factors of Frost et al.'s model are considered to reflect perfectionistic striving,

115 whereas concern over mistakes, doubts about actions, and parental concerns and expectations reflect

116 perfectionistic concerns (although some have suggested that these parental dimensions are more

117 closely linked to the development of perfectionism as opposed to being core aspects of

118 perfectionistic concerns, see Stoeber \& Otto, 2006). Moreover, such a dimensional approach allows

119 for distinctions to be made between different "types" of perfectionists: "healthy" perfectionists, who

120 are high in perfectionistic striving and low in perfectionistic concerns; and "unhealthy"

121 perfectionists, who are high in both perfectionistic striving and perfectionistic concerns (Hamachek,

122 1978; Stoeber \& Otto, 2006). In general, the literature surrounding these broad dimensions of

123 perfectionism suggests that perfectionistic striving (i.e., high personal standards) in the absence of

124 evaluative concerns is related to positive outcomes including: confidence (Hall et al., 1998; Koivula

125 et al., 2002), performance (Cox et al., 2002; Gould, Dieffenbach, \& Moffet, 2002), and the use of

126 task oriented coping (Gaudreau \& Antl, 2008). However, a combination of both perfectionistic

127 striving and perfectionistic concerns (i.e., unhealthy perfectionism) is somewhat more maladaptive,

128 being related to variables including neuroticism (Parker, 1997), anxiety (Koivula et al., 2002),

129 burnout (e.g., Gould, Udry, Tuffey, \& Loehr, 1996), and depression (e.g., Rice \& Mirzadeh, 2000).

130 Although researchers have yet to examine whether perfectionism is specifically related to

131 the 'yips', a consideration of some of the major issues involved in the 'yips' (e.g., anxiety) and

132 characteristics displayed by 'yips'-affected athletes (e.g., obsessive thinking) suggests that such a

133 relationship could exist. For example, across a range of populations perfectionism has been found to

134 be a consistent predictor of anxiety. Specifically, perfectionistic concerns (such as concern over 
135

mistakes) have been shown to be related to pre-performance anxiety in college students and athletes (e.g., Frost \& Henderson, 1991; Hall et al., 1998). In addition, unhealthy perfectionists report higher levels of state and trait anxiety than healthy perfectionists (e.g., Gotwals, Dunn, Causgrove Dunn, \& Gamache, 2010; Koivula, et al., 2002). As "unhealthy” perfectionists set high standards and are highly self-critical, they are likely to experience anxiety in response to situations that others would find less stressful (Frost \& DiBartolo, 2002). Because anxiety is a central component in the 'yips' experience, the positive association between anxiety and some specific components of perfectionism may make the 'yips' more likely.

Perfectionism is also related to obsessive thinking: the concern over mistakes and doubts about actions subscales are consistent predictors of obsessive-compulsive behaviors (e.g., Frost et al., 1990; Frost \& Steketee, 1997). Furthermore, researchers have also demonstrated positive relationships between personal standards and obsessive-compulsive behaviors (Frost et al., 1990; Rheaume, Freeston, Dugas, Letarte, \& Ladouceur, 1995). Thus it would appear that obsessivecompulsive individuals display characteristics that are consistent with unhealthy perfectionism (i.e., perfectionistic striving and perfectionistic concerns). Considering these findings alongside the reported obsessive nature of 'yips'-affected athletes (e.g., Smith et al., 2000), it seems likely that perfectionism (particularly unhealthy perfectionism) will be prominent in the development of the ‘yips'.

$$
\text { Consequently, the aim of the present study was to assess whether sportspeople with }
$$
perfectionist tendencies were more likely to suffer from the 'yips'. More specifically, given the links presented earlier between perfectionism, anxiety, and obsession, we expected that both perfectionistic strivings (personal standards and organization) and perfectionistic concerns (concern over mistakes and doubts about actions) would predict whether an individual suffered from the 'yips'. However, we did not include parental expectations and concerns in our investigation given our adult sample and the fact that some authors (e.g., Stoeber \& Otto, 2006) have questioned the 
160

161

162

163

164

165

166

167

168

169

170

171

172

173

relevance of these aspects of perfectionism to perfectionistic concerns. In summary, we expected the 'yips' to be associated with unhealthy perfectionism.

\section{Method}

\section{Participants}

Following institutional ethics approval, 120 male participants volunteered to take part in the study. The sample consisted of 60 participants $(n=20$ golfers, $n=20$ darts players, $n=20$ cricketers, $M$ age $=42.0 \mathrm{yrs}, S D=10.20$ ) who suffered from the 'yips' and 60 sport-matched participants $(M$ age $=43.0 \mathrm{yrs}, S D=9.40)$ who had never suffered from the problem. We selected golf, cricket and darts as previous investigations (e.g., Bawden \& Maynard, 2001; Smith et al., 2003; Rotheram et al., 2006; White, 1993) have identified that the 'yips' are particularly prevalent in these sports. Participants in both groups were randomly sampled (see procedures section) and had approximately 10 years playing experience at competitive league level or equivalent ('yips' group $M=10.1$ years, $S D=6.1$; non-'yips' group $M=10.3, S D=5.8$ ). All participants gave their informed consent to take part in the study.

\section{Measures}

Perfectionism. We used the shortened version (Cox et al., 2002) of Frost et al.'s. (1990)

Multidimensional Perfectionism Scale (FMPS). The shortened FMPS is a 22-item questionnaire assessing five dimensions of perfectionism: Concern over Mistakes (COM); Doubts about Actions (DAA); Parental Pressures (PP; a composite of parental expectations and parental pressures from the original Frost scale); Personal Standards (PS); and Organization (Org). Each item is scored on a 1 (strongly disagree) to 5 (strongly agree) scale, with higher scores indicating more perfectionism.

The shortened FMPS has been shown to be a valid measure of perfectionism in non-clinical samples, and was used over the original due to its improved factorial validity (see Cox et al., 2002). In the present study all subscales apart from the PP subscale were used. Composite reliabilities (CR's) were used to assess the internal consistency of each subscale of the shortened FMPS instead 
185

of Cronbach's alpha. The limitations of using Cronbach's alpha as a measure of internal consistency have existed in the literature for some time (e.g., Cortina, 1993), with recent commentaries (e.g., Sijitsma, 2009), further emphasising the limited use of Cronbach's alpha. For example, Cronbach's alpha is known to be inflated by scale length (Cortina, 1993). In addition, Cronbach's alpha assumes equal factor loadings for item subscales, which is rarely achieved in reality. Originating in the structural equation modelling literature, assessment of CR is considered superior to Cronbach's alpha as CR's do not assume equal factor loadings for each item, and are less likely to be affected by scale length (Fornell \& Larcker, 1981). The use of CR as a measure of internal consistency is gaining popularity within sport and exercise psychology as evidenced by its use in recent manuscripts (e.g, Silva et al., 2010; Williams \& Cumming, 2011). CR's in excess of .70 are considered acceptable (Fornell \& Larcker, 1981). In the present study CR's ranged from .71 to .86 (see Table 1).

Although perfectionism researchers (e.g., Stoeber \& Stoeber, 2009) recommend the use of domain specific measures of perfectionism, we chose to measure perfectionism at a general level as a result of previous research (e.g., Frost et al., 1990; Frost \& Steketee, 1997; Hall et al., 1998) providing strong evidence of the FMPS being related to key variables in the 'yips' process (namely anxiety and obsessive thinking). Thus we felt that the FMPS would be an appropriate instrument to use in this instance.

\section{Procedure}

'Yips' group. Participants in the 'yips' group were recruited from Rotheram et al.'s (2006) database of 'yips'-affected participants in golf, cricket, and darts. All individuals in the database had responded to an online mixed-methods survey regarding experiences of the 'yips'. While the full details of this database can be found in Rotheram et al., an overview is provided here for readers less familiar with this work. 
Rotheram et al. (2006) undertook an exhaustive literature review of the extant 'yips'

210 literature (particularly the initial questionnaires used by Smith and colleagues, 2000, 2003 to

211 identify 'yips' affected golfers), as well as a survey of British Association of Sport and Exercise

212 Sciences (BASES) accredited Sport Psychologists who reported having worked with individuals

213 suffering from the 'yips', to identify key factors involved in the 'yips' process. This information

214 was then used to develop the survey. The survey initially required individuals to read an

215 information sheet describing the 'yips' (described as "a long term severe breakdown of a skill that

216 could previously be performed with ease"), to provide sport specific demographic data, as well as

217 their perceptions of the aetiology of the 'yips'. Next, a range of tick box options were provided so

218 that participants could select the physical (e.g., jerk, tremor, freezing, tensing of affected muscles,

219 stomach sinking, tingling in hands and feet, chest pain, trembling, inability to physically perform, or

220 other) and psychological symptoms (e.g., self-focus, distraction, dizziness, feelings of unreality,

221 lack of control, fear, embarrassment, anxiety, external concerns, disorientation, or other) that

222 occurred when experiencing the 'yips'. Participants then completed open ended dialogue boxes

223 relating to their first experience of the 'yips', as well as providing a general description of what

224 happened during 'yips' situations.

Initial pilot testing of this measure was conducted to establish face validity on a small

number of individuals who were known to be 'yips' sufferers. All individuals in this pilot group had

experienced a severe breakdown in a skill which they could previously perform with ease for at

least 12 months. Based on feedback from the pilot group changes were made to the survey to

229 increase the readability of the items. Following these changes the survey was posted on-line so that

230 participants were able to self-select themselves into the survey. Once surveys were completed,

231 responses were scrutinized in order to ensure that only individuals who actually suffered from the

232 'yips' were entered into the database. More specifically, responses were examined for the

233 occurrence of at least one of the following criteria (chosen on the basis of previous 'yips' research, 
e.g., Bawden \& Maynard, 2001; Smith et al., 2003): an inability to perform a certain part of the sport that could previously be performed with ease; an extreme fear of performing; an extreme lack of control over the skill being performed; a long term movement problem; and the experience of a jerk, tremor, twitch or trembling. Thirty-five participants who had completed the survey failed to report at least of one these characteristics, and so Rotheram et al. deemed that these individuals were suffering from a poor performance, and did not have the 'yips'. On removal of these participants, 228 participants were entered into the database.

Participants in the database were contacted about the study by the second author to confirm that the 'yips' was still present. Of those participants who expressed an interest, stratified random sampling, based on sport type, was then used to obtain the final sample of 60 participants. We only used males as there were not enough females within the database to comprise a reasonable sample. The 60 participants were then sent the shortened FMPS via email or post and they returned their completed questionnaires to the second author.

Non-'yips' group. Selection criteria for the non-'yips' group were developed by the second, third, and fourth authors based on previous investigations into the 'yips' (e.g., Smith et al., 2003). Inclusion criteria were that the participant: had never experienced a physical disruption of any skill in their main sport or subsidiary sport played, had never been diagnosed with any form of movement disorder, had never had a close family member suffer from any form of movement disorder, and had played competitively in a high standard league (for darts players and cricketers) or equivalent (handicap of $<6$ for golfers) for at least 5 years. The second author made phone calls to local golf clubs, darts organizations and cricket clubs to advertise the project as well as placing advertisements in club houses and competition rooms. As with the 'yips' group, of those participants who expressed an interest in the study, stratified random sampling, based on sport type, was used to obtain the final sample of 60 participants. These participants were either sent a copy of the shortened FMPS along with a stamped addressed envelope, or completed the shortened FMPS at 
their sporting venue in small groups of no more than five individuals. Participants were asked to complete the measures without conferring with others and were assured of the confidentiality of their responses.

\section{Results}

\section{Preliminary analyses}

Descriptive statistics and Pearson correlations can be found in Table 1. Correlations between FMPS subscales were significant ( $r$ 's $=.24-.66$ ) with the exception of the Org-COM correlation. Bonferroni adjusted $t$-tests revealed that FMPS subscale scores were all higher in the 'Yips' group (all $p$ 's $<.005$ ). To examine whether sport type influenced scores on the perfectionism scales we performed a one-way MANOVA with sport type as the independent variable and the four perfectionism scales as the dependent variables. Box's M test for equality of Covariance matrices was non-significant $(p>.13)$ and the MANOVA revealed no multivariate effect for sport type, Wilks' $\lambda=.95, F(8,228)=0.89, p=.60$, partial $\eta^{2}=.03,1-\beta=.37$. Consequently the data were collapsed across sport type.

\section{Main analysis}

We used full model logistic regression to examine which aspects of perfectionism would predict the likelihood of an individual suffering from the 'yips'. Individuals in the 'yips' group were coded 1, and the non-'yips' group were coded 0 . Tests of multicollinearity were within acceptable limits, thus no variables were removed. The full model was significant, $\chi^{2}(4)=90.15, p<.001$, indicating that the FMPS subscales, as a set, were able to predict group membership (i.e., the 'yips' and non-'yips' group). The full model correctly classified $89 \%$ of participants as either 'yips' sufferers or non- 'yips' sufferers. In order to examine the impact of each of the FMPS subscales we inspected Wald statistics, odds ratios (and associated 95\% confidence intervals) and regression coefficients. A significant Wald test indicates that the independent variable reliably predicts the outcome. Odds ratios highlight the change in odds of being in a particular outcome category when 
the independent variable increases by one; odds ratios greater than one reflect an increase in odds of an outcome of 1 (being 'yips' affected in this case). Odds ratios can also be interpreted in terms of effect sizes, with odds ratios close to 1 indicating a small effect. Finally, regression coefficients are simply natural logs of the odds ratios and provide an indication of the direction of the relationship between the independent and dependent variable (in this case positive values reflect a greater likelihood of being 'yips' affected). The interested reader is referred to Tabachnick and Fidell (2007) where an excellent coverage of logistic regression is provided. Table 2 displays regression coefficients, Wald statistics, odds ratios and 95\% confidence intervals for odds ratios. Inspection of the Wald statistics indicated that only PS, Org, and COM were significant (positive) predictors of being 'yips'-affected.

\section{Discussion}

The aim of this study was to examine if perfectionism could predict whether an individual would suffer from the 'yips'. We hypothesized that 'yips'-affected individuals would be associated with high levels of both perfectionistic strivings and perfectionistic concerns (i.e., unhealthy perfectionism). Results provided some support for the hypothesis, as Personal Standards (PS), Organization (Org) and Concern Over Mistakes (COM) were all significant predictors of being 'yips'-affected. To the best of our knowledge, this is the first study to examine specific personality characteristics in relation to the 'yips' in sport. In general, the results imply that 'yips' sufferers display a pattern of perfectionism that is consistent with the unhealthy aspect of this personality variable. Thus, our results contribute to the emerging literature on the potential benefits and costs of particular aspects of perfectionism (e.g., Stoeber \& Otto, 2006), where striving for perfection coupled with harsh personal criticism can lead to profound performance difficulties. Although the results provide some support for the hypothesis, a more detailed examination of the data reveals three issues that warrant discussion: the nature of the mean perfectionism values for both groups; 
why COM was the only aspect of perfectionistic concerns to predict the 'yips'. We deal with these issues in the sections that follow.

Despite the fact that the 'yips' group reported significantly higher perfectionism scores than

313 the non-'yips' group, the actual mean scores for both groups were very low in comparison to values

314 reported in previous sport and mainstream psychology perfectionism studies ${ }^{1}$ (e.g., Rice \&

315 Mirzadeh, 2000; Sapieja, Dunn, \& Holt, 2011). Although this result is unexpected, and the values 316 presented rather atypical of sportspeople, we believe that the most parsimonious explanation is due 317 to perfectionism being measured at a general level as opposed to being measured at a domain 318 specific level. Although we felt that a general measure of perfectionism, the FMPS, would be 319 appropriate in this study (as the FMPS has been shown to predict, amongst other things, anxiety and 320 obsessive thinking), scores on generic measures of perfectionism are often lower than scores on 321 domain specific measures of perfectionism (e.g., Dunn, Gotwals, \& Causgrove Dunn, 2005) and so may not present a true picture of how perfectionistic the individual is in relation to the domain under investigation. With this in mind, future studies into perfectionism and the 'yips' may wish to consider the use of sport specific perfectionism measures alongside general measures of perfectionism to ensure that individual differences in perfectionism are more fully assessed.

On a somewhat related note, the mean values reported for the 'yips' group might lead one to question whether the 'yips' group actually displayed a pattern of healthy perfectionism² ${ }^{2}$ (because the actual mean values suggest relatively high PS and Org values coupled with relatively moderate COM and DAA) as opposed to unhealthy perfectionism. However, we believe that it is appropriate 330 to suggest that 'yips' sufferers display unhealthy perfectionism. First, the 'yips' group were 331 significantly higher on all facets of perfectionism (including COM and DAA) than the non-'yips' 332 group. Second, and more importantly, given that the main purpose of the study centred on 333 prediction, the absolute levels of each perfectionism subscale are less relevant than whether they 334 were able to predict the 'yips'. From the regression analysis it is clear that COM (along with PS and 
335 Org) predicted the 'yips'. Despite the fact that COM had the lowest odds ratio of the significant

336 predictors, COM contributed to the regression model in the presence of PS and Org, thus

337 accounting for a unique percentage of group membership that was not accounted for simply by PS

338 and Org. This suggests to us, at least, that the 'yips' is predicted by a combination of perfectionistic

339 striving and perfectionistic concerns (i.e., unhealthy perfectionism). However, given that this is the

340 first study to examine relationships between perfectionism and the 'yips', future research that is

341 able to confirm these effects (especially with sport specific measures of perfectionism) would be

342 extremely worthwhile.

343 While we believe that the 'yips' is associated with unhealthy perfectionism, it is somewhat

344 surprising that COM was the only aspect of perfectionistic concerns to predict the 'yips', and that

345 DAA failed to contribute. Perhaps the most parsimonious explanation centers on the differences

346 between COM and DAA. In the original conceptualization of perfectionism by Frost et al. (1990),

347 DAA differs from COM in that DAA does not focus specifically on the (harsh) evaluation of

348 mistakes, whereas COM does. Rather, DAA is more concerned with a general sense of doubt about

349 what one does. With this in mind, it seems possible to suggest that with regards to the 'yips'

350 specifically, the obsessive thinking and behaviors that affected individuals demonstrate might be

351 more closely tied to COM than DAA. To expand, COM reflects something of an "all or nothing"

352 mentality: if something is not perfect it constitutes a failure (cf. Frost et al., 1990). Thus, having the

353 'yips' likely indicates a total failure of an athlete's current approach (the 'yips' is present so

354 whatever is currently being done is not working), and may help to explain why 'yips' sufferers

355 constantly obsess about trying to solve the problem (Smith et al., 2000). While this explanation has

356 some appeal, it remains somewhat speculative especially without any insight (such as a measure of

357 obsessive thinking and/or anxiety) into precisely what causes 'yips'-affected individuals to act as

358 they do. Studies that are able to shed light on this issue would be particularly informative. 
Despite these issues of complexity, the results from the present study do indicate that perfectionism is able to predict those that are more likely to suffer from the 'yips'. This study consequently opens up a number of potential research questions. First, longitudinal designs that can confirm the direction of casualty between perfectionism and the 'yips' are warranted. While we favor an interpretation suggesting that perfectionism (amongst other things) causes the 'yips' over one which suggests that the 'yips' might lead to perfectionism (indeed from a theoretical standpoint we cannot see why having the 'yips' should increase one's level of perfectionism) our crosssectional design precludes us from being able to confirm this. Once causality can be established, the precise mechanisms underlying these effects also require attention. For example, given the relevance of anxiety and obsessive thinking in relation to perfectionism and the 'yips', it is conceivable that one, either, or indeed both, of these variables could act as mediators within the perfectionism-'yips' relationship. However, the role of anxiety within this relationship may depend on the type of 'yips' being suffered from (cf. Smith et al., 2003). Regardless, future research that is able to elucidate precisely why perfectionists are more likely to experience the 'yips' is warranted. Another avenue worthy of investigation is to examine the role of significant life and sportspecific events in the perfectionism-'yips' relationship. In their study of 'yips'-affected cricketers, Bawden and Maynard (2001) found that significant sport-related events (e.g., arguments with teammates, a dropped catch, first game for a new team) occurred immediately prior to the onset of the first experience of the 'yips'. Similarly, more recent research by Rotheram et al. (2006) has found that 'yips'- affected individuals often report the occurrence of major life events (e.g., death of parent, relationship breakdown, moving house) prior to the initial onset of the 'yips'. Perfectionists are known to be more susceptible to the negative consequences of significant life events (e.g., Hewitt, Flett, \& Ediger, 1996), and also react more strongly to these stressors, as the occurrence of a stressor exacerbates their perceptions of failure (Flett, Hewiit, \& Dyck, 1989). It may be that the obsessive nature of "unhealthy" perfectionists, coupled with the negative consequences of critical 
self-evaluation may result in the significant event having an unusually profound effect, resulting in the event becoming deep rooted. Theories of dissociation or conversion disorder (see Brown, 2004 for an overview) would suggest that psychological pain can manifest itself as physical symptoms in order to provide some relief from the psychological pain being experienced (e.g., in extreme cases, individuals with depression may self harm to gain some relief). Thus, it is conceivable that the 'yips' may be a result of the psychological pain associated with the life event. In this regard, significant life events may play a pivotal role in determining whether perfectionists experience the yips, and future research would do well to empirically examine this suggestion. a perfectionism perspective, researchers (e.g., Stoeber \& Stoeber, 2009) have suggested that domain-specific measures of perfectionism may be better predictors of outcomes than general perfectionism scales (such as the FMPS used in the present study). Indeed, sport specific measures of perfectionism have been recently developed and validated (e.g., Gotwals \& Dunn, 2009; Gotwals et al., 2010), and so would be worthy of consideration in future studies of perfectionism and the 'yips'.

Another issue for future research to explore is how best to assess the 'yips'. Although the measure in the present study has previously received initial support for its construct validity, in terms of being able to discriminate between true 'yippers' and those experiencing only a poor performance, the self-report nature of this measure means (as with all self-report instruments) that accuracy of participant responses remains open to question. Further to this, a consideration of whether individuals are suffering from different "types" of 'yips' was not included. More recently, 405 researchers have employed other methods of assessing the 'yips' in order to overcome some of the 406 limitations of survey based designs. For example, Bell, Skinner, and Fisher (2009) recorded the number of observable tremors or flinches in the dominant hand during golf putting, while Stinear and colleagues (2006) assessed EMG activity during putting as well as administering behavioral 
measures that are sensitive to focal hand dystonia. While the use of these behavioral and

410 observational measures may add some benefit over and above the sole reliance on questionnaires,

411 such measures may not capture all aspects of the 'yips' phenomenon (e.g., observation provides no

412 information as to the underlying causes of the 'yips'). Thus a combination of survey, observation,

413 and behavioral measures may provide the most comprehensive approach at this time.

414 Notwithstanding the aforementioned limitations, a number of applied implications emerge

415 from the present research. First, a consideration of perfectionist tendencies may help practitioners

416 and coaches to identify potential 'yippers' amongst their sportspeople. Second, given that elements

417 of unhealthy perfectionism predict the 'yips', it would seem that interventions aimed at reducing the

418 impact of perfectionistic concerns (and in the present context concerns over mistakes) would be

419 worthwhile. The use of solution-focused guided imagery (see Bell et al., 2009) may be particularly

420 useful in this regard. In addition, practitioners and coaches may wish to consider altering training

421 environments to incorporate mistakes into training, as such an approach may help to attenuate

422 perfectionists' worry associated with making mistakes.

In summary, this exploratory study indicates that perfectionism predicts the likelihood of

424 being affected by the 'yips'. The nature of the relationship is such that the 'yips' appears most likely 425 when individuals display characteristics associated with unhealthy perfectionism (i.e., striving for 426 perfection while simultaneously having concerns about achieving perfection). Future research 427 examining the potential mechanisms underlying these effects, as well as strategies that may reduce 428 the likelihood of the onset of the 'yips' for perfectionists would be worthwhile. 
Adler, C. H., Crews, D., Hentz, J. G., Smith, A. M., Mill, B., \& Caviness, J. N. (2005). Abnormal co-contraction in yips-affected but not unaffected golfers: Evidence for focal dystonia. Neurology, 64, 1813-1814.

Adler, C. H., Crews, D., Kahol, K., Santello, M., Noble, B., Hentz, J.,...Caviness, J. N. (2011). Are the Yips a Task-Specific Dystonia or “'Golfer's Cramp”? Movement Disorders, 26, 19931996.

Bawden, M. A. K., \& Maynard, I. W. (2001). Towards an understanding of the personal experience of the 'yips' in cricketers. Journal of Sports Sciences, 19, 937-953.

Brown, R. (2004). Psychological mechanisms of medically unexplained symptoms: an integrated perceptual model. Psychological Bulletin, 130, 793-812.

Cortina, J. M. (1993). What is coefficient alpha? An examination of theory and applications. Journal of Applied Psychology, 78, 98-104.

Cox, B. J., Enns, M. W., \& Clara, I. P. (2002). The multidimensional structure of perfectionism in clinically distressed and college student samples. Psychological Assessment, 14, 365-373.

Dunkley, D. M., Zuroff, D. C., \& Blankstein, K. R. (2003). Self-critical perfectionism and daily affect: Dispositional and situational influences on stress and coping. Journal of Personality and Social Psychology, 84, 234-252.

451 Dunn, J. G. H., Gotwals, J. K., \& Causgrove Dunn, J. (2005). An examination of the domain specificity of perfectionism among intercollegiate student-athletes. Personality and Individual Differences, 38, 1439-1448.

Flett, G. L., Hewitt, P. L., \& Dyck, D. G. (1989). Self-orientated perfectionism, neuroticism, and 
anxiety. Personality and Individual Differences, 10, 731-735.

Fornell, C., \& Larcker, D. F. (1981). Evaluating structural equation models with unobservable variables and measurement error. Journal of Marketing Research, 18, 39-50.

Frost, R. O., \& DiBartolo, P. M. (2002). Perfectionism, anxiety and obsessive-compulsive disorder. In Flett, G. L., \& Hewitt, P. L. (Eds). Perfectionism: Theory, Research and Treatment (pp. 341-371). American Psychological Association, Washington, DC.

Frost, R. O., \& Henderson, K. J. (1991). Perfectionism and reactions to athletic competition. Journal of Sport \& Exercise Psychology, 13, 323-335.

Frost, R. O., Marten, P., Lahart, C., \& Rosenblate, R. (1990). The dimensions of perfectionism. Cognitive Therapy and Research, 14, 449-468.

Frost, R. O., \& Steketee, G. (1997). Perfectionism in obsessive-compulsive disorder patients. Behaviour Research and Therapy, 35, 291-296.

Gaudreau, P., \& Antl, S. (2008). Athletes' broad dimensions of dispositional perfectionism: Examining changes in life satisfaction and the mediating role of sport-related motivation and coping. Journal of Sport \& Exercise Psychology, 30, 356-382.

Gotwals, J. K., \& Dunn, J. G. H. (2009). A multi-method multi-analytic approach to establishing internal construct validity evidence: The Sport Multidimensional Perfectionism Scale 2. Measurement in Physical Education and Exercise Science, 13, 71-92.

Gotwals, J. K., Dunn, J. G. H., Causgrove Dunn, J., \& Gamache, V. (2010). Establishing validity evidence for the sport multidimensional perfectionism scale-2 in intercollegiate sport. Psychology of Sport and Exercise, 11, 423-432.

Gould, D., Dieffenbach, K. \& Moffett, A. (2002). Psychological characteristics and their development in Olympic champions. Journal of Applied Sport Psychology, 14, 172-204.

Gould, D., Udry, E., Tuffey, S., \& Loehr, J. (1996). Burnout in competitive junior tennis players: I. A quantitative psychological assessment, The Sport Psychologist, 10, 322-340. 
Hall, H. K., Kerr, A. W., Matthews, J. (1998). Precompetitive anxiety in sport: the contribution of achievement goals and perfectionism. Journal of Sport \& Exercise Psychology, 20, 194-217.

Hamacheck, D. E. (1978). Psychodynamics of normal and neurotic perfectionism. Psychology, 15, 27-33.

Hewitt, P. L., \& Flett, G. L. (1991). Dimensions of perfectionism in unipolar depression. Journal of Abnormal Psychology, 100, 98-101.

Hewitt, P.L., Flett, G.L., \& Ediger, E. (1996). Perfectionism and depression: Longitudinal assessment of a specific vulnerability hypothesis. Journal of Abnormal Psychology, 105, 276-280.

Koivula, N., Hassmén, P., \& Fallby, J. (2002). Self-esteem and perfectionism in elite athletes: effects on competitive anxiety and self-confidence. Personality and Individual Differences, $32,865-875$.

McDaniel, K. D., Cummings, J. L., \& Shain, S. (1989). The 'yips': A focal dystonia of golfers. Neurology, 39, 192-195.

Parker, W. D. (1997). An empirical typology of perfectionism in academically talented children. American Educational Research Journal, 34, 545-562.

Rheaume, J., Freeston, M. H., Dugas, M. J., Letarte, H., \& Ladouceur, R. (1995). Perfectionism, responsibility and obsessive-compulsive symptoms. Behaviour, Research and Therapy, 33, 785-794.

Rice, K. G., \& Mirzadeh, S. A. (2000). Perfectionism, attachment, and adjustment. Journal of Counseling Psychology, 47, 238-250.

Rotheram, M., Bawden, M., Maynard, I., Thomas, O., \& Scaife, R. (2006). An exploratory investigation of the 'yips' across and within sport. In P. Hassmén \& N. Hassmén (Eds.), Yearbook 2006 from the Swedish Sport Psychology Association (pp. 1-22). Laholm, Sweden: Trydells Tryckeri AB. 
505 Rotheram, M., Thomas, O., Bawden, M., \& Maynard, I. (2007). Understanding the 'yips' in sport: A grounded theory interview study. Journal of Sports Sciences, 25, 323-324.

Sachdev, P. (1992). Golfer's cramp: clinical characteristics and evidence against it being an anxiety disorder. Movement Disorders, 4, 326-332.

Sapieja, K.M., Dunn, J. G. H., \& Holt, N. L. (2011). Perfectionism and perceptions of parenting styles in male youth soccer. Journal of Sport \& Exercise Psychology, 33, 20-39.

Sijtsma, K. (2009). On the use, the misuse, and the very limited usefulness of cronbach's alpha. Psychometrika, 74, 107-120.

Silva, M. N., Markland, D. Vieria, P. N., Coutinho, S. R., Carraça, E. V., Palmeira, A. L.,...Teixeira, P. J. (2010). Helping overweight women become more active: Need support and motivational regulations for different forms of physical activity. Psychology of Sport

Smith, A. M., Adler, C. H., Crews, D., Wharen, R. E., Laskowski, E. R., Barnes, K.,... Kaufman, K.R. (2003). The 'yips' in golf: continuum between a focal dystonia and choking. Sports Medicine, 33, 13-31.

Smith, A. M., Malo, S. A., Laskowski, E. R., Sabick, M., Cooney, W. P., Finnie, S. B.,...Kaufman, K. (2000). A multidisciplinary study of the 'yips' phenomenon in golf: an exploratory analysis. Sports Medicine, 30, 123-137.

Stinear, C. M., Coxon, J. P., Fleming, M. K., Lim, V. K., Prapavessis, H., \& Byblow, W. D. (2006). The yips in golf: Multimodal evidence for two subtypes. Medicine and Science in Sports \& Exercise, 11, 1980-1989.

Stoeber, J., \& Otto, K. (2006). Positive conceptions of perfectionism: Approaches, evidence and challenges. Personality and Social Psychology Review, 10, 295-319. 

Differences, 46, 530-535

531 Tabachnick, B. G. \& Fidell, L. S. (2007). Using multivariate Statistics (5 ${ }^{\text {th }}$ edition). Boston, USA: Allyn and Bacon.

533 White, J. (1993). Yikes it's golfers' 'yips'. Physician and Sports Medicine, 21, 13-14.

534 Williams, S. E., \& Cumming, J. (2011). Development and validation of the sport imagery ability 535 questionnaire. Journal of Sport \& Exercise Psychology, 33, 416-440. 


\section{Footnotes}

1. We would like to thank an anonymous reviewer for making this suggestion

2. We would like to thank the same anonymous reviewer for making this suggestion. 
$541 \quad$ Table 1

542 Descriptive statistics, zero-order correlations, and composite reliabilities for perfectionism

\begin{tabular}{ccccc}
\hline Variable & 1 & 2 & 3 & 4 \\
\hline 1. PS & - & & \\
2. Org & $.66^{* *}$ & - & \\
3. COM & $.24^{* *}$ & .08 & - & \\
4. DAA & $.35^{* *}$ & $.32^{* *}$ & $.45^{* * *}$ & - \\
Mean $(S D)$ 'Yips' group & $3.40(.73)$ & $3.42(.99)$ & $2.39(.80)$ & $2.66(.87)$ \\
Mean $(S D)$ Non-'yips' group & $2.29(.47)$ & $2.23(.48)$ & $2.04(.50)$ & $2.12(.55)$ \\
CR & .82 & .86 & .78 & .71 \\
\hline$* p<.05 ; * * p<.001$ & & &
\end{tabular}


559 Table 2

560 Logistic regression analysis for predicting 'yips' group status as a function of perfectionism

95\% confidence interval for Odds ratio

\begin{tabular}{cccccc} 
Variable & $B$ & Wald Chi-square & Odds ratio & Lower & Upper \\
\hline 1. PS & 2.16 & $14.43^{* *}$ & 8.70 & 2.85 & 26.56 \\
2.Org & 1.88 & $12.15^{* *}$ & 6.54 & 2.28 & 18.81 \\
3. COM & 1.17 & $4.04^{*}$ & 3.20 & 1.03 & 10.00 \\
4. DAA & .15 & .09 & 1.16 & 0.43 & 3.11 \\
Constant & -13.81 & $27.86^{* *}$ & & &
\end{tabular}

